\title{
Web Maps of Regional Attractiveness
}

\author{
Otakar Čerba $^{\mathrm{a},}{ }^{*}$, Jan Macura ${ }^{\mathrm{b}}$, Karel Charvát ${ }^{\mathrm{c}}$, Viktorie Sloupová ${ }^{\mathrm{a}}$ \\ ${ }^{a}$ University of West Bohemia, Plzeň, Czech Republic, cerba@kgm.zcu.cz,viktoris@students.zcu.cz \\ ${ }^{b}$ Wirelessinfo, Czech Republic, macurajan@gmail.com \\ c Plna4all, Czech Republic, charvat@ccss.cz \\ * Corresponding author
}

Keywords: Web map, Regional attractiveness, Thematic map, Clustering

\begin{abstract}
:
How attractive is this region? People ask this question very often. The main problem consists of the vagueness of the term "attractiveness" and the varied understanding and perception of the word "attractiveness". Attractiveness can be seen from the perspective of a farmer, a young man or woman, a businessman or a sociologist. The presented research is focused on providing information about regional attractiveness through interactive web maps. This approach is essential from many perspectives: (1) information presented as maps are much more attractive and intelligible than numbers or tables; (2) maps show information with spatial context; (3) web maps are the most accessible cartographic product; (4) interactive web maps enable to select the perspective of regional attractiveness based on users' requirements and preferences.
\end{abstract}

The development of maps is based on a data-driven approach following diverse statistical input datasets. The attractiveness is calculated as the index (values between 0 and 1 representing low or high attractiveness) or clusters (regions with similar regional attractiveness, which is not expressed by a number). Both methods consider various views on regional attractiveness by selecting input data and their weights in the calculation process. There are also two technological approaches. The first method starts with static maps developed in QGIS (or other GIS software). Such maps are published via an open QGIS plugin as a Web Map Service and uploaded to a map client. In regional attractiveness for African countries, Czech municipalities or European NUTS 3 regions, the client based on HSLayersNG (Figure 1) is used. These web maps are part of digital innovation hubs and enable simple interactivity such as zooming or drawing.

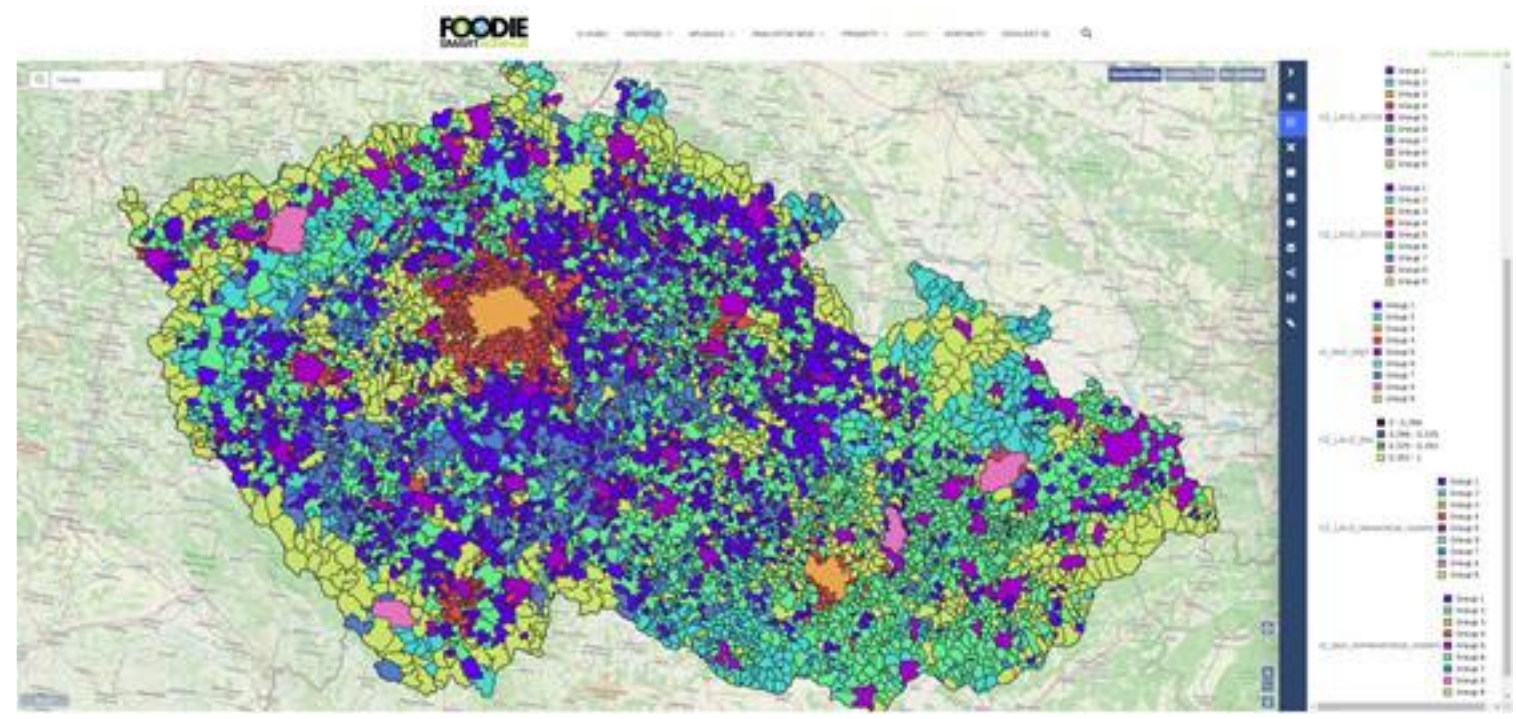

Figure 1. Map client on the digital innovation hub.

The second approach is more interactive than the first one. The web map application (Figure 2) is also based on the HSLayers-NG library, but it enables more user interactions. They can select input data sets, categories on input data or 
change the importance of particular inputs. Users can set up the perspective of regional attractiveness they prefer or need. Both technological approaches enable mixing maps of regional attractiveness with other data and creating sophisticated map compositions.

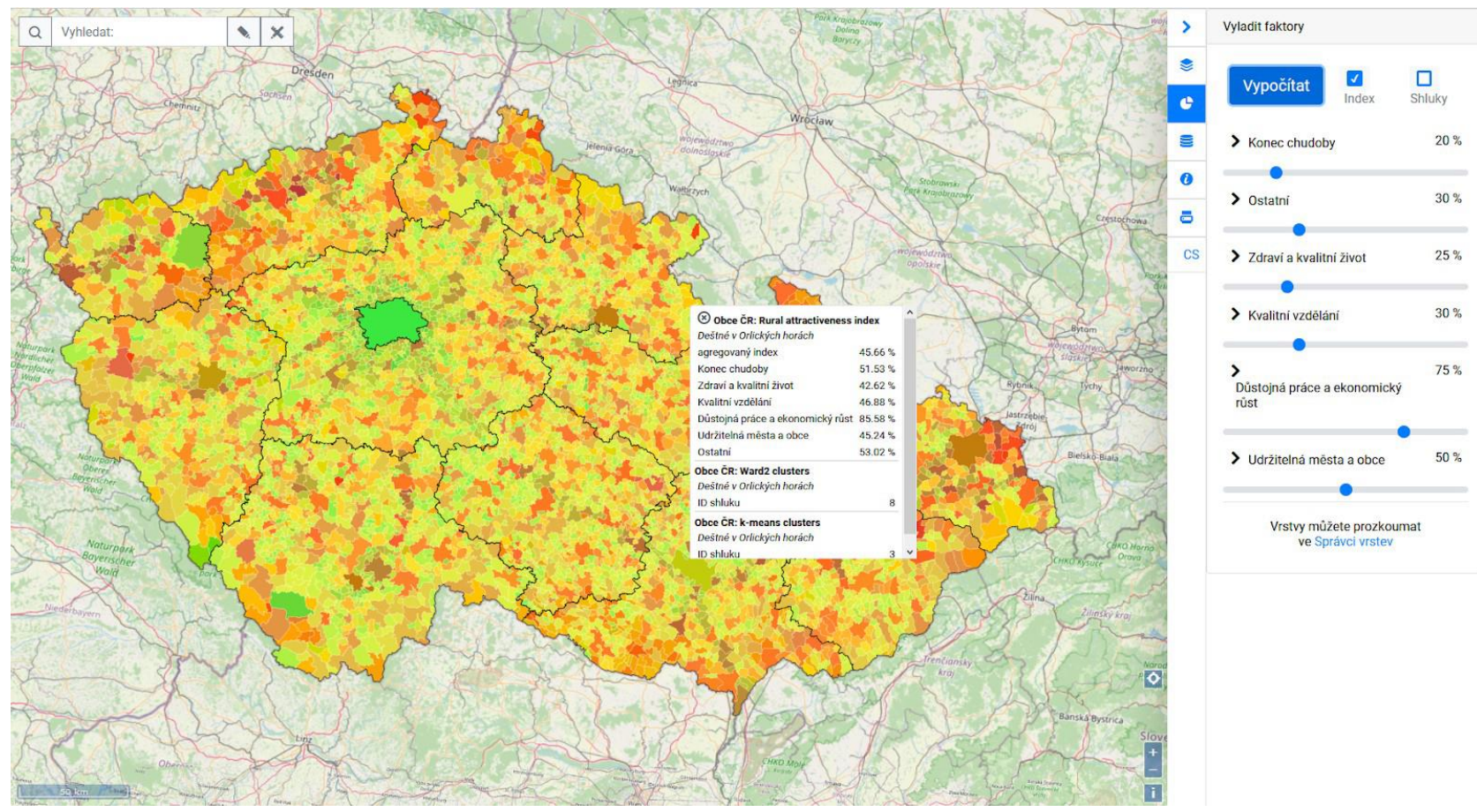

Figure 2. Web map application showing the index of rural attractiveness of Czech municipalities.

Nowadays, cartographic visualizations of regional attractiveness contain maps, assessments of regional attractiveness (clusters and indexes) and input data for African countries, Czech municipalities or European NUTS 3 regions. The solution is scalable, and therefore, it is possible to prepare similar interactive web maps for any purposes, requirements and needs. 